\title{
AVIFAUNAL DIVERSITY OF NIHARI TAWI RIVER IN DISTRICT RAJOURI, JAMIMU AND KASHMIR UT, INDIA
}

\section{Gurdeep Kumar Lecturer in Environmental Sciences (J\&K Govt.). Dr. Ranjan Sharma \\ Research Associate in INTACH.}

An avifaunal survey was conducted at river Nihari Tawi, district Rajouri in Jammu and Kashmir for one and half year,
starting from November 2015 to April 2017 . The study reveals a rich avian diversity and a total of 122 species of birds
belonging to 50 families were identified. As the water of the Nihari Tawi rich in dissolved oxygen and supports variety of
aquatic fishes makes it suitable for birds and attracts many migratory birds in the particular region during the winters.
Continuous monitoring of the avifaunal diversity is required to evaluate the ecological status of the birds and their
habitats. Birds plays an important role in maintaining the ecological balance. Due to increase in urbanization and various
anthropogenic activities, diversity and distribution of bird's species is on declining trend all over the globe. Therefore, it
is our prime concern to conserve the avifaunal diversity for maintaining the nature's balance.

\section{INTRODUCTION}

Avifaunal diversity is one of the most essential ecological indicators to evaluate the quality of habitats. Now a days, diversity of bird's species has been decreasing due to various anthropogenic activities, rapid increase in urbanization and random destruction of natural habitats by cutting nesting trees and using plants for commercial use of woods and lands. Thus, many species of birds may be forced to make their habitat in the urban areas and urge them to breed there. Birds are found throughout the world, at approximately all altitudes and in nearly every climate. Understanding the diversity and structure of bird communities is essential to delineate the importance of regional or local landscapes for avian conservation. Moreover, seasonal monitoring is very important to trace the dynamic movement of birds in various habitats. Water birds have attracted the attention of the public and researchers because of their beauty, abundance, visibility and social behavior, as well as for their recreational and economic importance. Recently, water birds have become of interest as indicators of water quality and as parameters of restoration success and regional biodiversity.

Birds may be influenced by various geographical conditions (Karr, 1976).Water bodies such as wetlands, ponds, lakes, etc. are important conservation site due to rich biodiversity and are among the most productive ecosystems worldwide. They harbor many globally threatened species (Green, 1996; Petrie, 1998). Diverse wetland complex are of greatest value in providing habitat for aquatic bird species (Miller, 2003). The avifauna is important for the ecosystem as they play various roles as scavenger, pollinators and predators of insect pest (Padmavati, et.al., 2010)

During the last few decades considerable studies on avifaunal diversity from different water bodies of India have been carried out by researchers like Singh (1929), Ali (1932), Davidar (1985), Ghazi (1962), Mujumdar (1984), Ghosal (1995) andWadatkar and Kasambe (2002).

Sohil and Sharma (2019) recorded 207 bird species belonging to 63 families in and around Jammu (J\&K); Malik and Sheikh (2020) recorded 64 species of birds belonging to 34 families in Tehsil Gool, district Ramban (J\&K).

\section{STUDY AREA}

District Rajouri is one of the 10 districts in Jammu division of Jammu and Kashmir (UT) and is divided into thirteen Tehsils (13). This stretch of Nihari Tawi lies between tehsil Siot and Kalakote situated at $33^{\circ} 06 \quad 40.37 \quad \mathrm{~N}$ to $33^{\circ} 12 \quad 59.73 \quad \mathrm{~N}$ latitude and $74^{\circ} 22 \quad 30.73 \quad E$ to $74^{\circ} 29 \quad 46.25 \quad E$ longitude with an elevation ranging from 500-1000 m a.m.s.l.
This river is fed by intermittent streams in form of nallas and khads. This area is surrounded by mixed pine forest and the scrubby hills.

\section{MATERIALS AND METHODOLOGY}

The study was conducted for a period of one and half year by using binocular, Nikon DSLR/ Canon EOS 700D camera, etc. which was later verified with the available literature on avian diversity such as Grimmet et. al., 1999; Salim Ali, 2002 and Grewal et. al.,2016.

Bird's identification is bit challenging process as they are very active/ energetic. Quick eye spotting is required in order to get a detail of the particular bird species. Recognition of birds is done by observing their movement, feeding habits, habitats, specific voice calls, shape, size, etc.

\section{RESULTS AND DISCUSSION}

There are total 122 bird species were identified belonging to 50 families during the study period from November 2015 to April 2017. The identified avian diversity is listed below in Table 1.

Table 1: List of Avifaunal diversity found in the study area

\begin{tabular}{|l|l|l|l|}
\hline $\begin{array}{l}\text { S. } \\
\text { No. }\end{array}$ & Family & Scientific Name & Common Name \\
\hline 1 & Accipitridae & Milvus migrans & black kite \\
\hline 2 & Accipitridae & Accipiter nisus & $\begin{array}{l}\text { euracian sparrow } \\
\text { hawk }\end{array}$ \\
\hline 3 & Accipitridae & Accipiter badius & shikra \\
\hline 4 & Accipitridae & Buteo buteo & common buzzard \\
\hline 5 & Accipitridae & $\begin{array}{l}\text { Neophron } \\
\text { percnopterus }\end{array}$ & egyptian vulture \\
\hline 6 & Accipitridae & $\begin{array}{l}\text { Pernis } \\
\text { ptilorhynchus }\end{array}$ & oriental honey buzzard \\
\hline 7 & Accipitridae & Gyps bengalensis & white rumped vulture \\
\hline 8 & Accipitridae & $\begin{array}{l}\text { Gyps } \\
\text { himalayensis }\end{array}$ & himalayan griffon \\
\hline 9 & Accipitridae & Elanus axillaris & white shouldered kite \\
\hline 10 & Alaudidae & Galerida cristata & crested lark \\
\hline 11 & Alcedinidae & $\begin{array}{l}\text { Megaceryle } \\
\text { lugubris }\end{array}$ & crested kingfisher \\
\hline 12 & Alcedinidae & $\begin{array}{l}\text { Halcyon } \\
\text { smyrnensis }\end{array}$ & $\begin{array}{l}\text { white throted } \\
\text { kingfisher }\end{array}$ \\
\hline 13 & Apodidae & $\begin{array}{l}\text { Tachymarptis } \\
\text { melba }\end{array}$ & alpine swift \\
\hline 14 & Ardeidae & Ardeola grayii & indian pond heron \\
\hline 15 & Ardeidae & Egretta garzetta & little egret \\
\hline
\end{tabular}


PARIPEX - INDIAN JOURNAL OF RESEARCH | Volume - 10 | Issue - 10 | October - 2021 | PRINT ISSN No. 2250 - 1991 | DOI : $10.36106 /$ paripex

\begin{tabular}{|c|c|c|c|c|c|c|c|}
\hline 16 & Ardeidae & Bubulcus ibis & cattle egret & \multirow[t]{2}{*}{57} & \multirow[t]{2}{*}{ Hirundinidae } & \multirow{2}{*}{$\begin{array}{l}\text { Petrochelidon } \\
\text { fluvicola }\end{array}$} & \multirow{2}{*}{$\begin{array}{l}\text { streak throated } \\
\text { swallow }\end{array}$} \\
\hline 17 & Ardeidae & Ardea cinerea & grey heron & & & & \\
\hline 18 & Ardeidae & Ardea purpurea & purple heron & 58 & Laniidae & Lanius schach & long tailed shrike \\
\hline 19 & Bucerotidae & Ocyceros birostris & Indian grey hornbill & 59 & Leiothrichidae & Leiothrix lutea & red billed leiothorix \\
\hline \multirow{2}{*}{20} & \multirow{2}{*}{ Certhiidae } & \multirow{2}{*}{$\begin{array}{l}\text { Certhia } \\
\text { himalayana }\end{array}$} & \multirow{2}{*}{$\begin{array}{l}\text { bar tailed tree } \\
\text { creeper }\end{array}$} & 60 & Leiothrichidae & Argya caudata & common babbler \\
\hline & & & & \multirow[t]{2}{*}{61} & \multirow[t]{2}{*}{ Leiothrichidae } & \multirow{2}{*}{$\begin{array}{l}\text { Trochalopteron } \\
\text { lineatum }\end{array}$} & \multirow{2}{*}{$\begin{array}{l}\text { streaked } \\
\text { laughingthrush }\end{array}$} \\
\hline \multirow[t]{2}{*}{21} & \multirow[t]{2}{*}{ Cettidae } & \multirow{2}{*}{ Horornis fortipes } & \multirow{2}{*}{$\begin{array}{l}\text { brown flanked bush } \\
\text { wabler }\end{array}$} & & & & \\
\hline & & & & \begin{tabular}{|l|}
62 \\
\end{tabular} & Leiothrichidae & Turdoides striata & jungle babbler \\
\hline 22 & Charadriidae & $\begin{array}{l}\text { Vanellus } \\
\text { malabaricus }\end{array}$ & $\begin{array}{l}\text { yellow wattled } \\
\text { lapwing }\end{array}$ & 63 & Megalaimidae & $\begin{array}{l}\text { Psilopogon } \\
\text { haemacephalus }\end{array}$ & coppersmith barbat \\
\hline 23 & Charadriidae & Vanellus indicus & red wattled lap wing & 64 & Megalaimidae & $\begin{array}{l}\text { Psilopogon } \\
\text { asiaticus }\end{array}$ & blue throted barbet \\
\hline \multirow[t]{2}{*}{24} & \multirow[t]{2}{*}{ Cisticolidae } & Orthotomus & common tailor bird & 65 & Megalaimidae & Psilopogon virens & great barbet \\
\hline & & & & 66 & Meropidae & Merops orientalis & green bee eater \\
\hline 25 & Cisticolidae & Prinia socialis & ashy prinia & 67 & Monarchidae & Terpsiphone & Indian paradise \\
\hline 26 & Cisticolidae & Prinia hodgsonii & grey brested prinia & 01 & & paradisi & flycatcher \\
\hline 27 & Cisticolidae & Prinia crinigera & himalayan prinia & 68 & Motacillidae & Motacilla & white browed \\
\hline 28 & Columbidae & Spilopelia & spotted dove & & & maderaspatensis & wagtail \\
\hline & & chinensis & & 69 & Motacillidae & Motacilla citreola & citrine wag tail \\
\hline 29 & Columbidae & Streptopelia & red collared dove & 70 & Motacillidae & Motacilla cinerea & grey wagtail \\
\hline & & tranquebarica & & 71 & Muscicapidae & Rhyacornis & plumbeous water \\
\hline 30 & Columbidae & Columba livia & rock dove & & & fuliginosa & redstart \\
\hline 31 & Coraciidae & $\begin{array}{l}\text { Coracias } \\
\text { benghalensis }\end{array}$ & indian roller & 72 & Muscicapidae & $\begin{array}{l}\text { Myophonus } \\
\text { caeruleus }\end{array}$ & $\begin{array}{l}\text { blue whistling } \\
\text { thrush }\end{array}$ \\
\hline 32 & Corvidae & Corvus splendens & house crow & 73 & Muscicapidae & Calliope & himalayan ruby \\
\hline 33 & Corvidae & Corvus & large billed crow & & & pectoralis & \\
\hline & & macrorhynchos & & 74 & Muscicapidae & Eumyias & verditer flycatcher \\
\hline 34 & Corvidae & Corvus & jungle crow & & & & \\
\hline $0 \pm$ & Uiviuae & culminatus & jurgie ciow & 75 & Muscicapidae & $\begin{array}{l}\text { Copsychus } \\
\text { saularis }\end{array}$ & $\begin{array}{l}\text { oriental magpie } \\
\text { robin }\end{array}$ \\
\hline 35 & Corvidae & $\begin{array}{l}\text { Dendrocitta } \\
\text { vagabunda }\end{array}$ & ree pie & 76 & Muscicapidae & $\begin{array}{l}\text { Saxicoloides } \\
\text { fulicatus }\end{array}$ & indian robin \\
\hline 36 & Corvidae & $\begin{array}{l}\text { Dendrocitta } \\
\text { formosae }\end{array}$ & grey treepie & 77 & Muscicapidae & $\begin{array}{l}\text { Chaimarrornis } \\
\text { leucocephalus }\end{array}$ & $\begin{array}{l}\text { white capped } \\
\text { redstart }\end{array}$ \\
\hline 37 & Corvidae & Garrulus & black headed jay & 78 & Muscicapidae & Saxicola caprata & pied bushchat \\
\hline & & lanceolatus & & 79 & Muscicapidae & Oenanthe fusca & brown rock chat \\
\hline 38 & Cuculidae & Hierococcyx & common hawk cuckoo & \begin{tabular}{|l|}
80 \\
\end{tabular} & Muscicapidae & Ficedula tricolor & slaty blue flycatcher \\
\hline & & varius & & 81 & Muscicapidae & Monticola & blue rock thrush \\
\hline 39 & Cuculidae & Cuculus canorus & common cuckoo & & 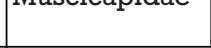 & solitarius & \\
\hline 40 & Cuculidae & $\begin{array}{l}\text { Cuculus } \\
\text { micropterus }\end{array}$ & indian cuckoo & 82 & Nectariniidae & $\begin{array}{l}\text { Aethopyga } \\
\text { siparaja }\end{array}$ & crimson sun bird \\
\hline 41 & Cuculidae & Taccocua & sirkeer malkoha & 83 & Nectariniidae & Cinnyris asiaticus & purple sun bird \\
\hline & & leschenaultii & & 84 & Paradoxornithi & Chrysomma & yellow eyed \\
\hline 42 & Cuculidae & Eudynamys & asian koel & & dae & sinense & babbler \\
\hline & & scolopaceus & & 85 & Paridae & Parus cinereus & cinereous tit \\
\hline 43 & Cuculidae & $\begin{array}{l}\text { Centropus } \\
\text { sinensis }\end{array}$ & greater coucal & 86 & Passeridae & $\begin{array}{l}\text { Passer } \\
\text { domesticus }\end{array}$ & house sparrow \\
\hline 44 & Dicruridae & $\begin{array}{l}\text { Dicrurus } \\
\text { macrocercus }\end{array}$ & black drongo & 87 & Passeridae & $\begin{array}{l}\text { Passer } \\
\text { cinnamomeus } \\
\end{array}$ & russet sparrow \\
\hline \begin{tabular}{|l|}
45 \\
\end{tabular} & Dicruridae & $\begin{array}{l}\text { Dicrurus } \\
\text { leucophaeus }\end{array}$ & ashy drongo & 88 & Pellorneidae & $\begin{array}{l}\text { Pellorneum } \\
\text { ruficeps }\end{array}$ & puff throted babbler \\
\hline 46 & Dicruridae & $\begin{array}{l}\text { Dicrurus } \\
\text { hottentottus }\end{array}$ & hair crested drongo & 89 & $\begin{array}{l}\text { Phalacrocoraci } \\
\text { dae }\end{array}$ & $\begin{array}{l}\text { Phalacrocorax } \\
\text { fuscicollis }\end{array}$ & indian cormorant \\
\hline \begin{tabular}{|l|}
47 \\
\end{tabular} & Emberizidae & Emberiza lathami & crested bunting & 90 & Phasianidae & Ortygornis & grey francolin \\
\hline 48 & Emberizidae & Emberiza stewarti & white capped bunting & & & pondicerianu & \\
\hline 49 & Estrildidae & & scally brested munnia & 91 & Phasianidae & Gallus gallus & red jungle fowl \\
\hline 49 & Estrilatae & punctulata & scany orested munna & 92 & Phasianidae & $\begin{array}{l}\text { Lophura } \\
\text { leucomelanos }\end{array}$ & kalij pheasant \\
\hline 50 & Estrildidae & Euodice & indian silverbill & 93 & Phasianidae & Pavo cristatus & Indian Peafowl \\
\hline 51 & Estrildidae & malabarica & scaly breasted munia & 94 & Phylloscopidae & $\begin{array}{l}\text { Phylloscopus } \\
\text { xanthoschistos }\end{array}$ & $\begin{array}{l}\text { grey hooded } \\
\text { warbler }\end{array}$ \\
\hline & & punctulata & & 95 & Phylloscopidae & & humes leaf wabler \\
\hline 52 & Falconidae & Falco tinnunculus & asian kestral & & & humei & \\
\hline $53 \mid$ & Fringillidae & Chloris spinoides & $\begin{array}{l}\text { yellow breasted } \\
\text { greenfinch }\end{array}$ & 96 & Phylloscopidae & $\begin{array}{l}\text { Phylloscopus } \\
\text { collybita }\end{array}$ & common chiffchaff \\
\hline 54 & Fringillidae & $\begin{array}{l}\text { Carpodacus } \\
\text { erythrinus }\end{array}$ & common rose finch & 97 & Picidae & \begin{tabular}{|l|}
$\begin{array}{l}\text { Dendrocopos } \\
\text { nanus }\end{array}$ \\
\end{tabular} & $\begin{array}{l}\text { brown capped } \\
\text { pygmy woodpecker }\end{array}$ \\
\hline 55 & Hirundinidae & Hirundo rustica & barn swallow & 98 & Picidae & Dendrocopos & fulvous brested \\
\hline 56 & Hirundinidae & Riparia chinensis & grey throated martin & & & & \\
\hline
\end{tabular}




\begin{tabular}{|c|c|c|c|}
\hline 99 & Picidae & Leiopicus auriceps & $\begin{array}{l}\text { brown fronted wood } \\
\text { pecker }\end{array}$ \\
\hline 100 & Picidae & \begin{tabular}{|l} 
Dinopium \\
benghalense
\end{tabular} & $\begin{array}{l}\text { flameback wood } \\
\text { pecker }\end{array}$ \\
\hline 101 & Psittacidae & $\begin{array}{l}\text { Psittacula } \\
\text { cyanocephala }\end{array}$ & $\begin{array}{l}\text { plum headed } \\
\text { parakeet }\end{array}$ \\
\hline 102 & Psittaculidae & Psittacula finschii & $\begin{array}{l}\text { grey headed } \\
\text { parakeet }\end{array}$ \\
\hline 103 & Psittaculidae & Psittacula eupatria & alexandrine parakeet \\
\hline 104 & Psittaculidae & Psittacula krameri & rose ringed parakeet \\
\hline 105 & Pycnonotidae & Pycnonotus cafer & red vented bulbul \\
\hline 106 & Pycnonotidae & $\begin{array}{l}\text { Pycnonotus } \\
\text { goiavier }\end{array}$ & yellow vented bulbul \\
\hline 107 & Rallidae & Zapornia akool & \begin{tabular}{|l|l}
$\begin{array}{l}\text { brown crake } \\
\text { (jal kukdi) }\end{array}$ \\
\end{tabular} \\
\hline 108 & Rallidae & $\begin{array}{l}\text { Amaurornis } \\
\text { phoenicurus }\end{array}$ & \begin{tabular}{|l|} 
white-breasted \\
waterhen \\
\end{tabular} \\
\hline 109 & Rhipiduridae & $\begin{array}{l}\text { Rhipidura } \\
\text { albicollis }\end{array}$ & white throated fantail \\
\hline 110 & Scolopacidae & Tringa ochropus & green sandpiper \\
\hline 111 & Sittidae & $\begin{array}{l}\text { Sitta } \\
\text { cinnamoventris }\end{array}$ & $\begin{array}{l}\text { chestnut bellied } \\
\text { nuthatch }\end{array}$ \\
\hline 112 & Stenostiridae & $\begin{array}{l}\text { Culicicapa } \\
\text { ceylonensis } \\
\end{array}$ & $\begin{array}{l}\text { grey headed canary } \\
\text { flycatcher }\end{array}$ \\
\hline 113 & Strigidae & $\begin{array}{l}\text { Glaucidium } \\
\text { cuculoides }\end{array}$ & asian barred owlet \\
\hline 114 & Sturnidae & \begin{tabular}{|l|} 
Acridotheres \\
ginginianus
\end{tabular} & bank myna \\
\hline 115 & Sturnidae & $\begin{array}{l}\text { Acridotheres } \\
\text { fuscus }\end{array}$ & jungle myna \\
\hline 116 & Sturnidae & \begin{tabular}{|l|} 
Sturnia \\
pagodarum
\end{tabular} & brahminy starling \\
\hline 117 & Sturnidae & Acridotheres tristis & common myna \\
\hline 118 & Timaliidae & $\begin{array}{l}\text { Erythrogenys } \\
\text { erythrogenys }\end{array}$ & $\begin{array}{l}\text { rusty cheeked } \\
\text { scimitar babbler }\end{array}$ \\
\hline 119 & Troglodytidae & \begin{tabular}{|l|} 
Troglodytes \\
troglodytes
\end{tabular} & eurasian wren \\
\hline 120 & Turdidae & Turdus atrogularis & black throated thrush \\
\hline 121 & Upupidae & Upupa epops & euracian hoopoe \\
\hline 122 & Zosteropidae & \begin{tabular}{|l|} 
Zosterops \\
palpebrosus
\end{tabular} & oriental white eye \\
\hline
\end{tabular}

10. Malik, W.S and Sheikh, T. (2020). A Preliminary Checklist of Birds in Tehsil Gool of District Ramban, Jammu and Kashmir. Journal of Wildlfe Research, 8(2):10-19.

11. Miller, A.A. (2003). Influence of habitat characteristics on wetland birds abundance and species richness at Ordway Prairie, South Dakota. M.S. thesis, South Dakota state University. Ecology 80:1806-1816.

12. Mujumdar, N. (1984). On a collection of birds from Bastar district, M.P. Record Zoological survey of India, Occasional paper No. 59:54.

13. Padmavati, A., Alexandar R. and Anbarashan, M. (2010). Our Nature, 8: 247-253.

14. Petrie, S. A. (1998). Nutrient reserve dynamics, foraging strategies, molt whistling Ducks in South Africa. Ph. D. dissertation, University of the Witwatersrand Johannesburg, South Africa.

15. Singh, T.C.N. (1929). A note of the pollination of Erythring Indica by birds. Journal of Bombay Natural History Society, 33:960-462.

16. Sohil, A. and Sharma, N. (2019). A preliminary Survey of Bird Communities around Jammu, (Jammu \& Kashmir). Biological Forum - An International Journal, 11 (2): 27-49. Wadatkar, J.S. and Kasambe, R. (2002). Checklist of Birds from Pohara-Malkhed reserve forest, Distt. Amravati, Maharashtra. Zoological.Print Journal, 17(66):807-811.

\section{CONCLUSION}

It is concluded from the present study that the stretch along the Nihari Tawi River in District Rajouri has rich avifaunal diversity. There is also wide variety of plant and tree species which are present in the entire stretch of the study area may act as a suitable habitat for the avian diversity. Presently, this area is not much studied in view of avifaunal diversity and this study may highlighted the scope of avifaunal studies and helpful in conserving and maintaining the ecological balance. Further, it will plays a signi cant role in biodiversity documentation at the regional level, besides feeding into policy-making in the country.

\section{REFERENCES:}

1. Ali, S. (1932). Flowers birds and birds flower in India, Journal of Bombay Natural History Society, 35:573-605.

2. Ali, S. (2002). The book of Indian birds. Bombay Natural History Society, India.

3. Davidar, P. (1985). Ecological interactions between the mistletoes and their avian pollinators in south India, Journal of Bombay Natural History Society, 82:45-60.

4. Ghazi, H.K. (1962). Piscivorous birds of Madras, Madras Journal of fisheries, 1(1):106-107.

5. Ghosal, D.N. (1995). Avifauna of conservation areas, No. 7, Fauna of Kanha Tiger Reserve.Zoological survey of India (ZSI), pp 63-9l.

6. Green, A.J. (1996). Seasonal of globally threatened anatidae in relation to theats, distribution, migration patterns and habitat use. Conservation Biology, 10:1435- 1445 .

7. Grewal, B., Sen, S., Singh, S., Devasar, N. and Bhatia, G. (2016). A pictorial field guide to birds of India. Om Publications, India.

8. Grimmet, R., Inskipp, C. and Inskipp, T. (1999). Pocket guide to the birds of the Indian subcontinent. Oxford university press, Delhi.

9. Karr J.R. (1976). Seasonality resource availability and community diversity in tropical bird communities, American Naturalist, 105:423-435. 